\title{
【Transaction】
}

\section{Effects of Draw Ratio and Additive on Knot-Pull Breaking Phenomenon in a Polypropylene Monofilament}

\author{
Tatsuma Kunimitsu*1, Chisa Ikeda*1, Shuntaro Oshima*1, \\ Toshifumi Ikaga*1, KyoungHou Kim*1, Yutaka Ohkoshi*1,2,\#, \\ Masayuki Takata*3, and Tomoyoshi Yamashita*3 \\ ${ }^{* 1}$ Faculty of Textile Science and Technology, Shinshu University, 3-15-1 Tokida, Ueda, Nagano, 386-8567, Japan \\ ${ }^{* 2}$ Institute for Fiber Engineering, Shinshu University, 3-15-1 Tokida, Ueda, Nagano, 386-8567, Japan \\ ${ }^{* 3}$ Mitsubishi Chemical Corporation, 1-1-1 Marunouchi, Chiyoda-ku, Tokyo, 100-8251, Japan
}

\begin{abstract}
Knot-pull strength is as important as tensile strength, but the mechanism by which the fiber in a knot breaks has not been sufficiently elucidated. In the present study, the effects of the draw ratio and a meltkneaded additive on the knot-pull strength of polypropylene monofilament, that is, a thick single fiber, were discussed by comparing the tensile and loop strengths of the fiber. The knot deformation behavior during a knot-pull test was also investigated. In contrast to the increase in tensile strength at a high draw ratio, the knot-pull strength was hardly affected by the additive, and the loop strength was reduced by the additive. During a knot-pull test on a high-draw-ratio fiber, the knot thickness continued to decrease to breakage, even after the knot length stopped decreasing. This behavior indicates that the highly drawn fiber breaks at the buckled part in the knot owing to the lateral compressional forces.
\end{abstract}

(Received 1 September, 2020; Accepted 29 October, 2020)

\section{Introduction}

Knot-pull strength-the tensile breaking stress of a knotted fiber-is one of the most important fiber properties. It is often regarded as a more important property than tensile strength, particularly with regard to ships, fisheries, and civil engineering [1] Polypropylene (PP) monofilament, that is, a thick single fiber, is commonly used for these applications because it is highly flexible. The tensile strength of PP fibers has increased recently, but their knot-pull strength has barely increased. This sluggish increase in the knot-pull strength has now become a critical problem.

The sluggish increase may be attributed to the complex breakage of knotted fibers. For example, the draw ratio at which the knot-pull strength reached a maximum is often lower than the draw ratio of the tensile strength maximum [2-4]. This indicates that a knotted fiber is not only broken by the tensile force but also by other forces, such as bending, compressional, twisting, shearing, and frictional forces applied to the knotted fiber [5, 6]. Yamaki explained the knot breakage mainly by the tensile force under the influence of radial compressional force [1], Pieranski et al. explained the knot breakage with computer simulations mainly by the bending force [7], and Uehara et al. interpreted the influence of twisting force on breakage [8]. Because the mechanism by which knotted fiber breaks is complex, the fiber breaking behavior also varies depending on the crosssectional shape of the fiber or fiber bundle, the material, and the operating environment. The location of the fiber breakage varies, for example, most knotted fibers tend to break in the vicinity of the knot entrance [9], but fibers also break within the knot [3]. There have been many other studies on the breaking mechanism of knotted fibers. Konda et al. [10, 11] investigated the knot breaking mechanism using tensile stress-strain curves. They ignored the shearing and lateral compressional forces and assumed that the fiber breaks according to the sum of the tensile and bending strains. Yabe suggested that a knotted fiber is broken by the weakest of three

\# corresponding author: Yutaka Ohkoshi (E-mail: yokoshi@shinshu-u.ac.jp) 
factors: the sum of the bending and frictional forces, the tensile strength under the influence of the radial compressional force, and the bending force that causes fiber buckling [3]. Fiber breakage with buckling has also been reported by Uehara et al. [8], Uddin et al. [12], and Lemstra et al. [13].

A previous study has reported on the knot-pull strength improvement of PP fibers via melt blending of the polyethylene component [14]. The report explained the improvement by the network structure formed in the fiber. The knot-pull strength might be improved by slippage between incompatible components of polyethylene and PP. In the present study, we focused on the effects of an acrylic triblock copolymer additive on the knot-pull strength. This additive is usually used as the adhesive, compound, and resin modifier [15], and reportedly increases the toughness of epoxy resin compounds by more than 20 times [16]. Because of the low compatibility between the additive and PP [15], it is also expected that the additive improves the knot-pull strength. To investigate the effects of the additive and the draw ratio on the knot-pull strength of drawn PP monofilaments (thick PP single fibers), the knot-pull strength was compared with the tensile and loop strengths. The broken fiber ends following loop and knot-pull tests were examined using a scanning electron microscope (SEM). The deformation behavior of the knot was also investigated by image processing a video obtained during the knot-pull test.

\section{Experimental}

\subsection{Sample}

The PP monofilaments, that is, thick single fiber, used in this study were fabricated by melt-spinning and drawing. Fibers containing $5 \mathrm{wt} \%$ additive were also fabricated and compared with the neat PP fibers. The neat PP polymer used for the melt spinning was isotactic polypropylene of Prime Polypro ${ }^{\mathrm{TM}}$ (grade Y2000GV; melt index $=18 \mathrm{~g} / 10 \mathrm{~min}$ ) supplied by Mitsubishi Chemical Co. (Tokyo, Japan). The additive KURARITY $^{\mathrm{TM}}$ (comprising an methyl/n-butyl/methyl acrylic triblock copolymer; grade LA4285; melt index $=31 \mathrm{~g} / 10 \mathrm{~min}$ [17]) was also supplied by Mitsubishi Chemical Co. (Tokyo, Japan). The neat polymer was melt-extruded from a BT-30-S 2-42-L twin-screw extruder (Research Laboratory of Plastics Technology Co., Ltd., Tokyo, Japan) using a spinneret with a diameter of $0.7 \mathrm{~mm}$ and a length of $2.1 \mathrm{~mm}$. The spinning temperature, mass flow rate, and take-up speed were $220{ }^{\circ} \mathrm{C}, 1.5 \mathrm{~g} / \mathrm{min}$, and $100 \mathrm{~m} / \mathrm{min}$, respectively. The $5 \mathrm{wt} \%$ additive containing PP fiber was also fabricated under the same spinning conditions by mixing a $10 \mathrm{wt} \%$ additive containing masterbatch with the neat polymer.

The as-spun fibers were drawn continuously via the speed difference between the feed and take-up rollers. The fiber feed speed was fixed at $1 \mathrm{~m} / \mathrm{min}$. A hot plate heater with a contact length of $225 \mathrm{~mm}$ set to $140{ }^{\circ} \mathrm{C}$ was used for drawing. The maximum draw ratio was the draw ratio at which the fiber could be drawn stably for more than $10 \mathrm{~min}$. The drawing tension was measured using a tension meter (HS-1500 S, Eiko Sokki Co., Ltd., Osaka Japan) equipped with a 1 $\mathrm{N}$ pickup sensor, and the fiber diameter was measured using an outer diameter detector (LS-9006, Keyence Co., Ltd.). Both measurements were captured by a data logger (NR-2000, Keyence Co., Ltd.) every $100 \mathrm{~ms}$. The drawing stress was calculated from the obtained tension and fiber diameter.

\subsection{Thermomechanical property measurements}

Differential scanning calorimetry (DSC) was performed using a Thermo Plus DSC 8230 system (Rigaku Co., Ltd.) from room temperature to $200{ }^{\circ} \mathrm{C}$ at a rate of $10 \mathrm{~K} / \mathrm{min}$. Approximately $2 \mathrm{mg}$ of a cut fiber sample was enclosed in an aluminum pan. Nitrogen gas was supplied at a flow rate of $50 \mathrm{~mL} / \mathrm{min}$ during the measurement. The melting peak temperature $\left(T_{m}\right)$ and the difference between the heat of fusion and the heat of cold crystallization $\left(\Delta H_{m}\right)$ were determined from the DSC curve.

\subsection{Tensile test}

The tensile test, the loop test, and the knot-pull test were performed for the drawn fibers at room temperature using an Autograph AGS-X instrument (Shimadzu Co., Ltd.) equipped with a $50 \mathrm{~N}$ load cell and an air chuck. An overhand knot was selected for the knot-pull test. The tensile strength, loop strength, and knot-pull strength were determined accordingly. The elongation at break and the initial modulus were also determined by the tensile test. Their averages and standard deviations were calculated using at least 12 specimens. The initial crosshead distance was 40 $\mathrm{mm}$, and the elongation speed was $40 \mathrm{~mm} / \mathrm{min}$ for the drawn fibers. The initial modulus was calculated in the strain range of $0.1 \%-1.6 \%$. The fiber diameter $(d)$, which was used for all fiber strength calculations, was measured before each test with using a digital microscope (VHX-1000, Keyence Co., Ltd). The 
samples for the loop and knot-pull tests were prepared so that the loops and knots were located at the midpoints of the chuck.

The broken fiber edges after the loop and knotpull tests were examined using an SEM (VE9800/8800, Keyence Co., Ltd.). The knot deformation behavior during the knot-pull test was investigated using a digital microscope (VHX-1000, Keyence Co., Ltd), which was equipped with a video camera, and was set on an XYZ axis translation stage. The frame rate was 28 frames/s, and image resolution was $0.95 \mu \mathrm{m} /$ pixel. During the knot-pull test, the vertical location of the knot was kept in the image by adjusting the microscope height. Still images were extracted from the video using Motion Capture AVI (Digimo Co., Ltd.), and were analyzed using ImageHyper2 (Digimo Co. Ltd.).

\section{Results and discussion}

\subsection{Drawing}

The result from the DSC measurements on the as-spun fibers is presented in Table 1. Both samples had only one melting peak at $167^{\circ} \mathrm{C}$.

Table 1 Results for the as-spun fibers

\begin{tabular}{ccccc}
\hline Sample & $\begin{array}{c}\text { Additive } \\
\text { Content } \\
(\mathrm{wt} \%)\end{array}$ & $\begin{array}{c}\text { Fiber } \\
\text { Diameter } \\
(\mu \mathrm{m})\end{array}$ & $\begin{array}{c}T_{m} \\
\left({ }^{\circ} \mathrm{C}\right)\end{array}$ & $\begin{array}{c}\Delta H_{m} \\
(\mathrm{~J} / \mathrm{g})\end{array}$ \\
\hline Neat PP & 0 & $147 \pm 5$ & 167 & 76 \\
\hline Additive-PP & 5 & $147 \pm 9$ & 166 & 70 \\
\hline
\end{tabular}

The drawing stresses are presented in Fig. 1. The additive reduced the drawing stress at an equivalent draw ratio. It also increased the maximum draw ratio and the whitening draw ratio, at which

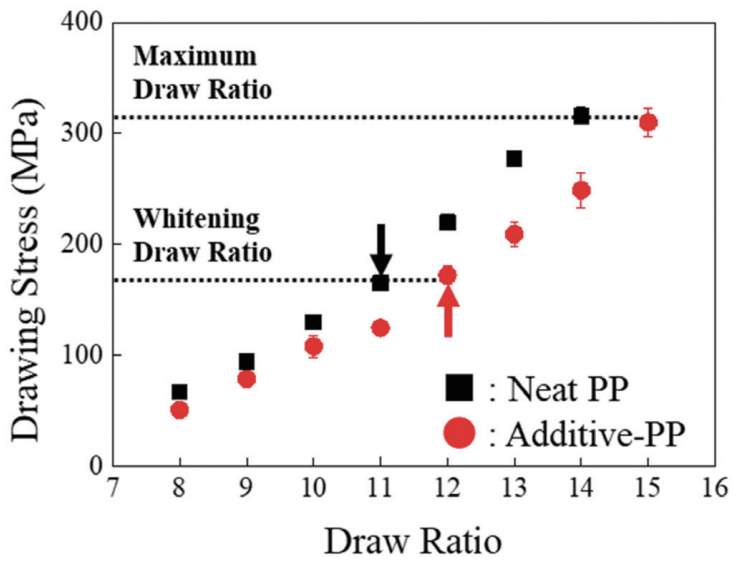

Fig. 1 Drawing stress versus draw ratio. The sample markers are defined in the figure, and their whitening draw ratios are indicated by arrows. The whitening and maximum drawing stresses are indicated by dotted lines. fiber whitening began. In contrast, the additive had little influence on the whitening stress and maximum drawing stress. These results indicate that the additive acted as a plasticizer in the fiber, that is, it promoted slippage between fibrils. The slippage between fibrils leveled the drawing stress applied to the fibrils, then the slippage increased the whitening draw ratio and the maximum draw ratio, whereas the tolerable maximum drawing stress and the whitening stress were not affected by the slippage.

\subsection{Mechanical properties}

Fig. 2 presents the tensile, loop, and knot-pull strengths, whereas Fig. 3 and Fig. 4 show the initial modulus and elongation at break, respectively. All the fibers were broken at the knot or loop part for each test. The tensile strength and the initial modulus increased, whereas the elongation at break decreased with the draw ratio. The tensile strength of the additive PP fiber increased monotonically up to the maximum draw ratio of the fiber, whereas the tensile

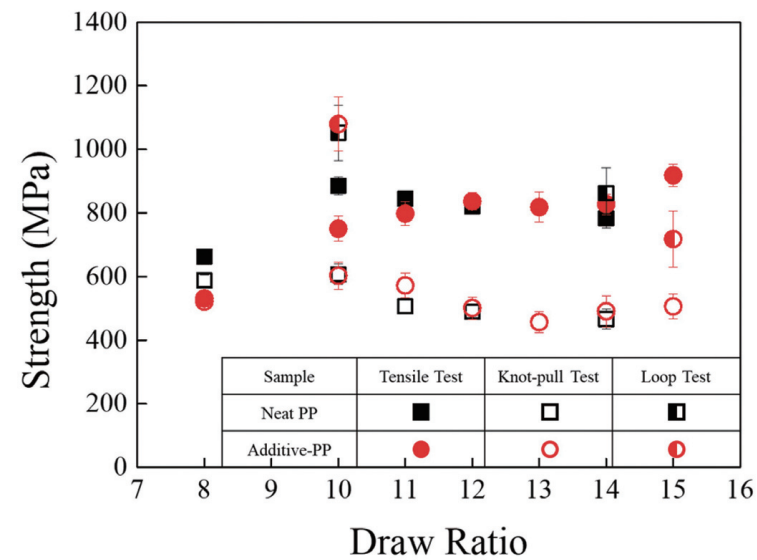

Fig. 2 Tensile, loop, and knot-pull strengths of the drawn fibers versus the draw ratio. The sample and test methods are represented in the figure.

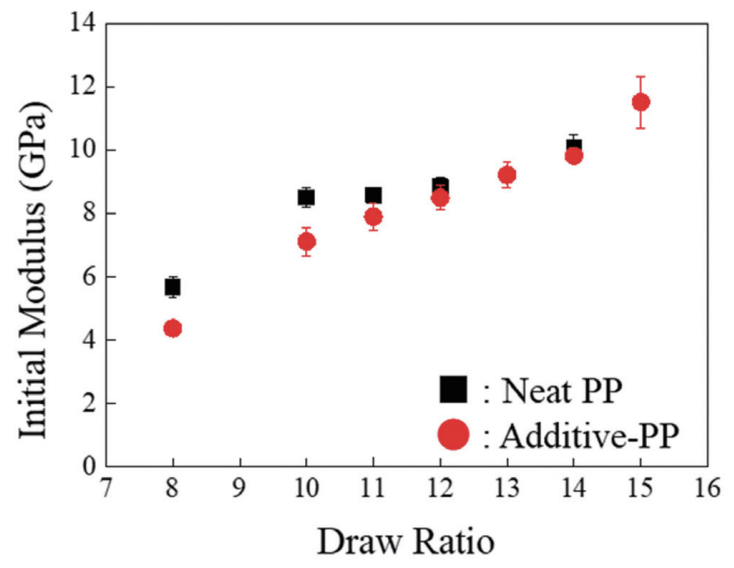

Fig. 3 Initial moduli of the drawn fibers versus the draw ratio. The samples are represented in the figure. 


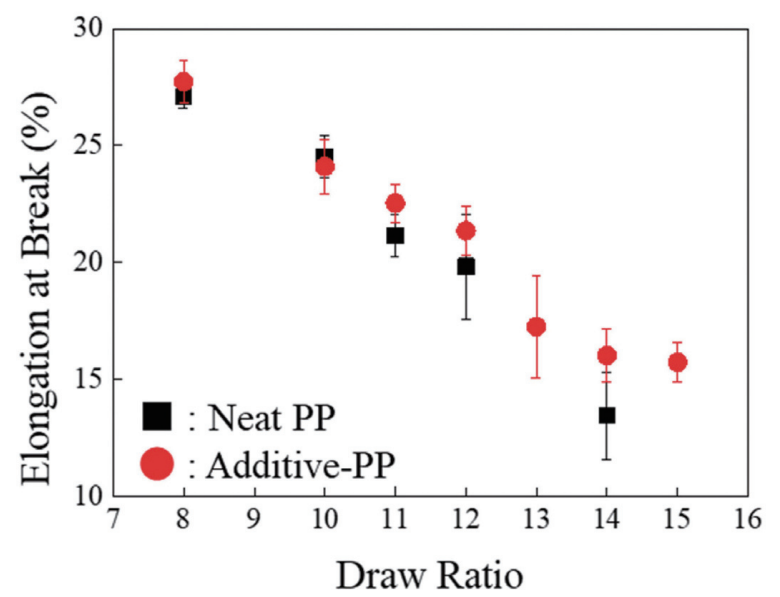

Fig. 4 Elongation at break of the drawn fibers versus the draw ratio. The samples are represented in the figure.

strength of neat PP fiber was almost saturated over a draw ratio of 10 . Furthermore, as with the increase in the maximum draw ratio, the additive increased the elongation at break for the fiber with the equivalent draw ratio. The loop strength was clearly larger than the tensile strength for a fiber drawn 10 times, while the loop strength was significantly reduced for the maximally drawn fibers. In particular, the loop strength of the additive-containing maximally drawn fiber was obviously lower than its tensile strength. In contrast to the loop strength, the knot-pull strength was constantly lower than the tensile strength over a draw ratio of 8 . Furthermore, the additive had no significant effect on the knot-pull strength. That is, although the additive increased the tensile strength of the highly drawn fibers, it did not increase their knot strength.

The additive increased the maximum draw ratio but had little effect on the whitening stress and maximum drawing stress. The additive also increased the tensile strength of the maximally drawn fiber but reduced the tensile strength of the fiber drawn at the lower draw ratio. Low compatibility between the additive and PP has been reported [15], as confirmed by the DSC results described in Section 3.1. Therefore the PP and additive phases should be separated in the fiber, and the additive-which is dispersed among the PP fibrils-is thought to be stretched by the elongational deformation applied in the spinning and drawing processes. The increase in the maximum draw ratio under the unchanged maximum drawing stress is thought to be caused by slippage at the additive / PP fibril interface with leveling of the stress applied to the fibrils. Stress leveling increased the tensile strength of the highly drawn fibers, whereas the defects formed by slippage at the interface reduced the tensile strength of the low-ratio drawn fibers, because fibrillation was observed at the breaking points of the additive-containing fibers, as shown below. In contrast to the low-ratio drawn fibers, the highly drawn fibers should be fibrillated when they break regardless of the additive. This is because the highly drawn fibers were already whitened by the macroscale voids formed among the fibrils [18]. The fibers should be fibrillated when they break owing to the development of the voids; then, the additive does not reduce the tensile strength of highly drawn fibers, but increases the tensile strength and elongation at break by leveling the stress applied to the PP fibrils.

Fig. 5 shows the SEM images in the vicinity of the fiber breaking point after the loop and knot-pull

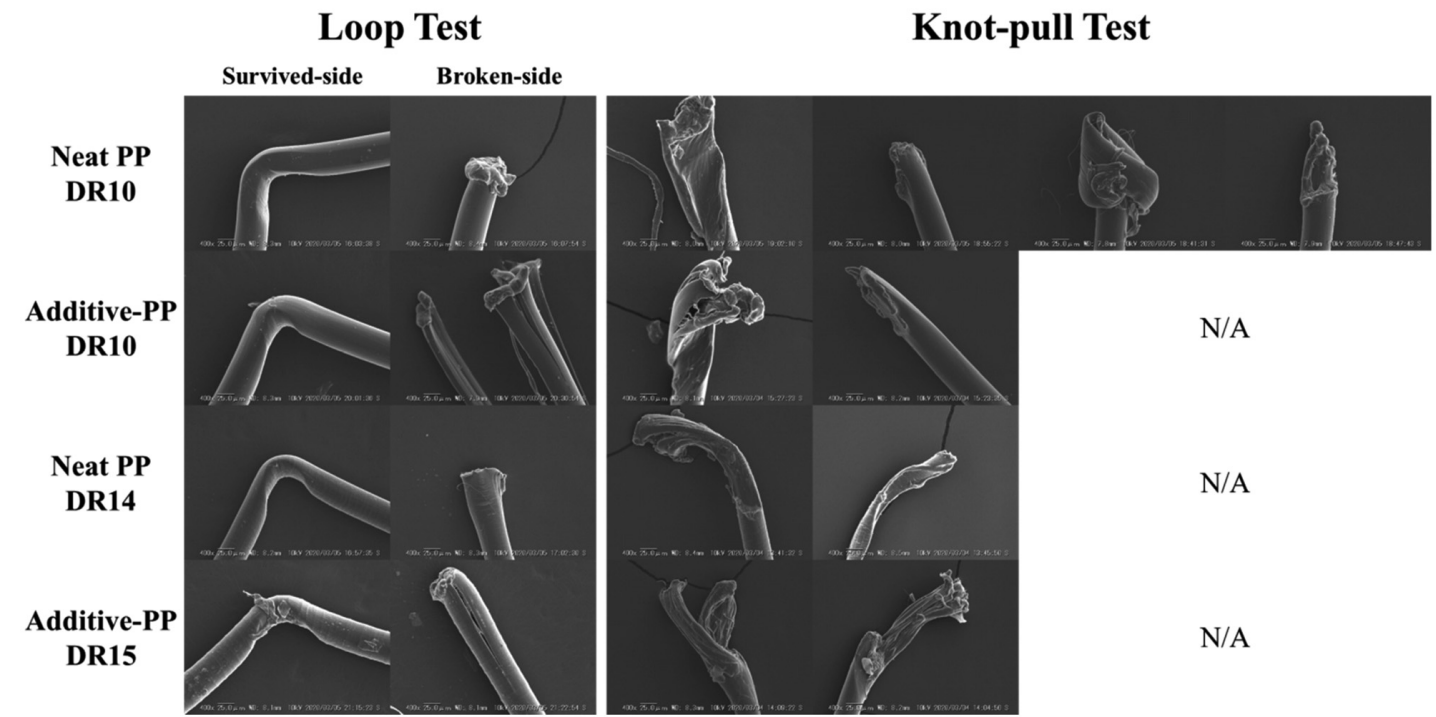

Fig. 5 Scanning electron microscope images in the vicinity of the fiber breaking ends and the surviving fiber after the loop and knot-pull tests. The sample and draw ratios are noted in the figure. 
tests. There was obvious lateral buckling in the surviving side of the fibers after the loop test, particularly in the maximally drawn fibers. However, it is unlikely that the fibers were broken by bending because there were no cracks or kinks perpendicular to the fiber axis on the outside or inside the fibers, respectively. Moreover, there was fibrillation in the broken-side fibers after the loop test, particularly in the additive-containing fibers. This suggests that the maximally drawn fibers were broken with fibrillation at the buckled part.

After the knot-pull test, only one broken end of the 10-times-drawn fiber was buckled, while the other end was not buckled. Furthermore, some knots of the 10-times-drawn PP fiber survived at the broken end. In contrast, both ends of the maximally drawn fibers were buckled. Moreover, the broken ends of the maximally drawn fibers, in particular the additivecontaining fibers, were fibrillated. These results suggest that the maximally drawn fibers were broken at the buckled bending part of the knot with fibrillation, as in the loop test, while the 10-timesdrawn fiber broke in the vicinity of the knot entrance.

\subsection{Deformation behavior of the knot}

As discussed in Section 3.2, there was a difference between the high-draw-ratio and low-drawratio fibers with regard to the fiber breaking mechanism in the knots. A video of the knot deformation behavior until fiber breakage was recorded during the knot-pull tests. The maximally drawn fibers and the 10-times-drawn fibers were selected to represent the high-draw-ratio and lowdraw-ratio fibers. The effects of the additive were also examined. The average knot-pull stress-strain curve for these samples were also compared with that of the

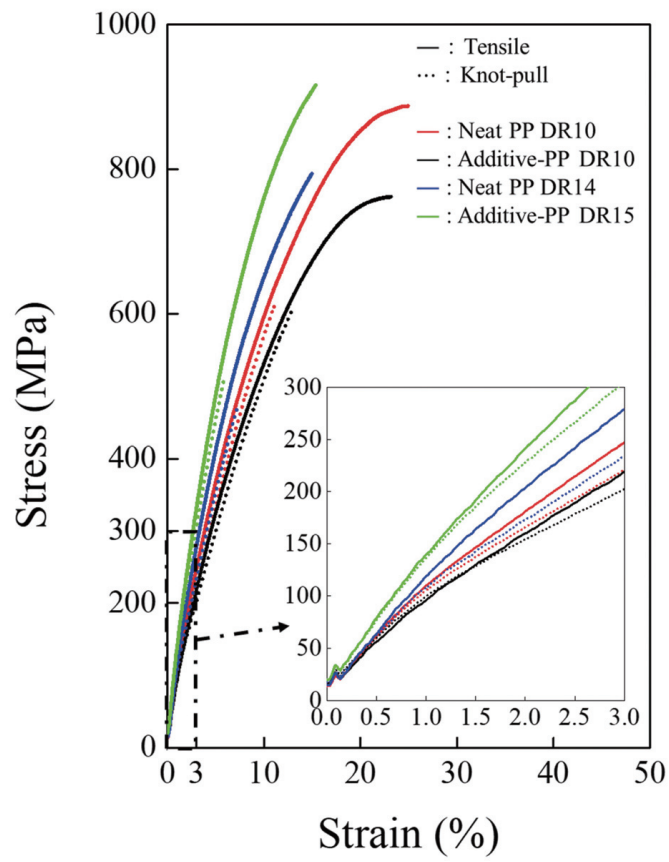

Fig. 6 Average stress-strain curve for the tensile (solid line) and knot-pull tests (dotted line). Both strains are defined as the ratio between the change and initial crosshead distance. Enlarged figure is also shown. The sample and draw ratio are noted in the figure.

tensile test, as shown in Fig. 6. For all samples, the knot-pull stress became lower than the tensile stress at a stress of $\sim 100 \mathrm{MPa}$. Fig. 7 shows still images extracted from the video. The knot seemed to tighten below an applied stress of $100 \mathrm{MPa}$, and subsequently shrank with increasing applied stress. These results suggested that the knot size decreased mainly by pulling out of the fiber from the knot below $100 \mathrm{MPa}$, and it decreased mainly by buckling of fiber in the knot above $100 \mathrm{MPa}$. The knot size of the low-drawratio fibers appeared to be larger than that of the high-draw-ratio fibers under an equivalent applied

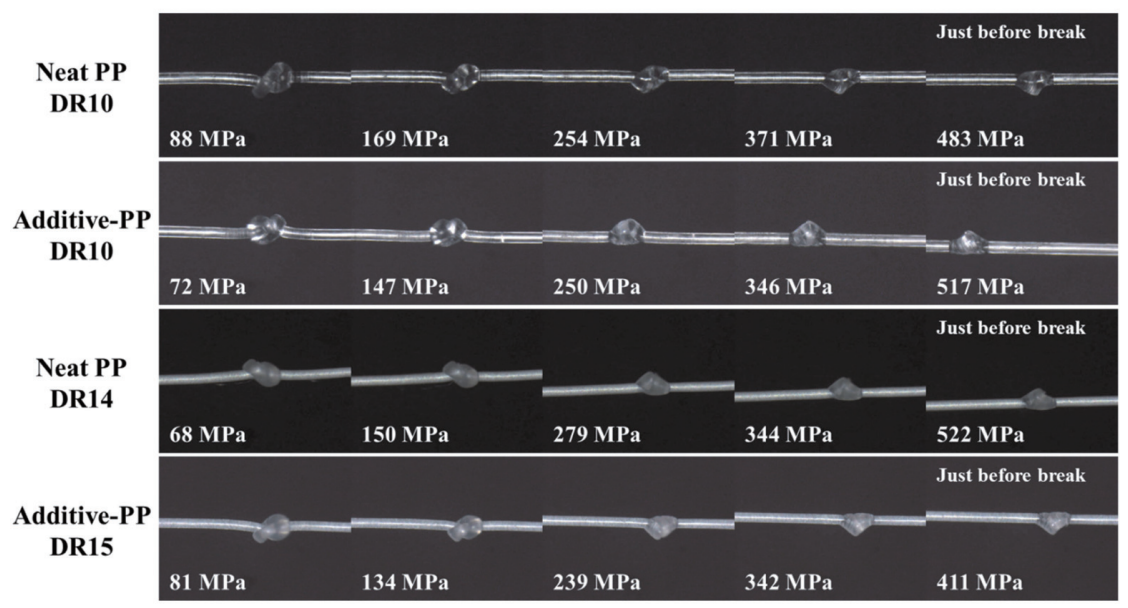

Fig. 7 Still images taken in the vicinity of the knot during the knot-pull test. The sample, draw ratio, and each applied stress are noted for each image. 
stress. Furthermore, in contrast to the round-shaped knots of the low-draw-ratio fibers, the knots of the high-draw-ratio fibers were somewhat elongated toward the horizontal direction. Both knot shapes became pentagonal under larger applied stresses. However, the change likely occurred at the lower applied stress in the knots of the high-draw-ratio fibers. The pentagonal knots of the high-draw-ratio fibers mainly shrank vertically with the increasing applied stress, whereas those of the low-draw-ratio fibers shrank omnidirectionally. Furthermore, fiber constriction at the knot ends was observed just before the break, which suggests that the radial compressional force was applied at the knot entrance.

As described above, the knot deformation behavior depended on the fiber sample, in particular the change in size and shape of its knot. Accordingly, we quantified the area, length, and thickness of each knot by applying image processing as follows. First, the diameter profile was obtained from the still image by binarization, blotting out the fiber part, and removing noise using a median filter. The threshold value used for binarization was unified for each test. Fig. 8 shows the typical diameter profile. As shown in the figure, it was possible to obtain the knot length $(l)$ along the fiber axis because there was a clear distinction between the diameter profiles of the knot and fiber. The knot area (a) was obtained by integrating the diameter profile in the range of $l$, and the average thickness of the knot was obtained by dividing $a$ by $l$. The normalized knot area $(A)$, the length $(L)$, and the thickness $(T)$ were calculated using equations (1) to (3) to cancel the effect of the fiber diameter $(d)$ :

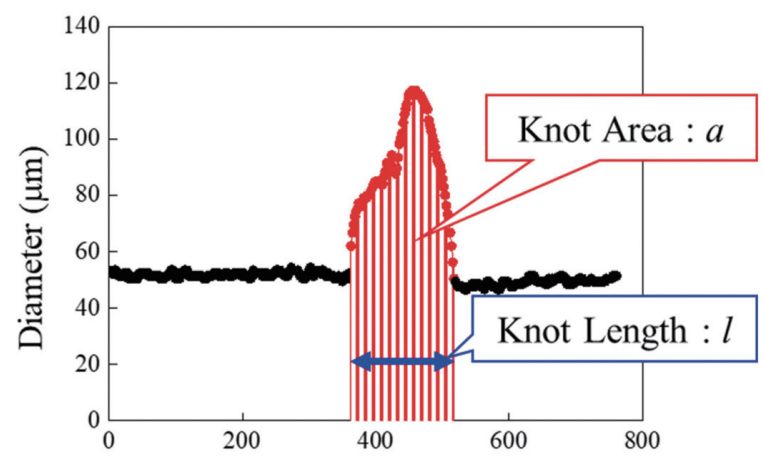

Length of Fiber Axis Direction $(\mu \mathrm{m})$

Fig. 8 Typical diameter profile in the vicinity of the knot (neat polypropylene (PP) fiber, draw ratio $10)$. The knot area $(a)$ and length $(l)$ are shown in the figure.

$$
\begin{aligned}
A & =\frac{a}{d^{2}} \\
L & =\frac{l}{d} \\
T & =\frac{A}{L}=\frac{a}{l d}
\end{aligned}
$$

Fig. 9 shows the normalized area $(A)$, the length $(L)$, and the average thickness $(T)$ of the knot. The smaller $T$ value of the high-draw-ratio fiber corresponds to the horizontally elongated knot shape, as mentioned above. $A, L$, and $T$ all decreased until the applied stress reached $200 \mathrm{MPa}$, which was almost equal to the applied stress at which the knot became pentagonal. At applied stresses over $200 \mathrm{MPa}$, all the decreases became gentle. However, in contrast to the $L$ and $T$ values of the low-draw-ratio fibers, which both decreased, in the high-draw-ratio fibers only $T$ decreased whereas $L$ did not. The decrease in only $T$ indicated the buckled bending part mainly deformed before breakage. Moreover, as observed in the lowdraw-ratio fibers, the decrease in $L$ seemed to be suppressed by the additive.

As demonstrated above, it was possible to quantify the deformation behavior of the knot by image processing, which enabled a clearer representation of the change to the knot shape observed in the videos. However, the shape of a knot cannot be sufficiently expressed by its length and thickness alone. For example, because the knot is cylindrically unsymmetrical, the $T$ value is necessarily affected by the imaging direction, but the $L$ value is not. Owing to the small trial number, there is some level of uncertainty, but there was no clear difference in reproducibility observed between $L$ and $T$ in the present study, which indicates that the imaging direction had little influence on reproducibility. More detailed analysis can be expected from a greater trial number and a higher image resolution.

\subsection{Fiber breaking mechanism of the knot}

Many kinds of force are applied to a knotted fiber, including tensile, bending, compressional, twisting, shearing, and frictional forces [5, 6]. Among them, the effects of twisting and shearing on knotted fiber breakage are reportedly small [3, 10]. Two dominant fiber breaking forms were observed for the overhand knot in the present study, that is, tensile breakage resulting from the normal stress difference between the tensile force and the radial compressional force, and fiber breakage with buckling and fibrillation resulting from the lateral compressional force (Fig. 10). That is, the low-draw-ratio fibers broke in the vicinity 


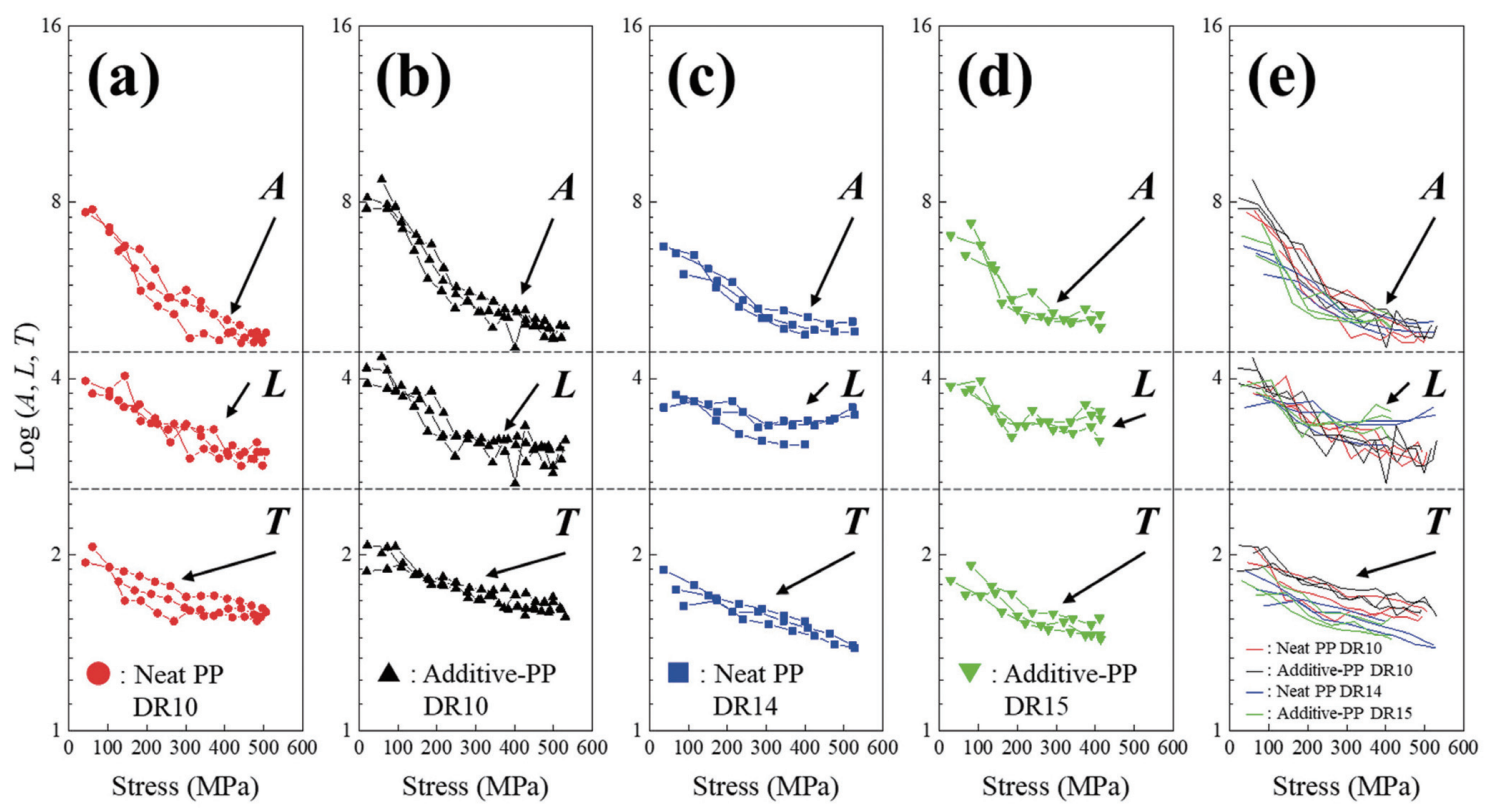

Fig. 9 Normalized knot area $(A)$, knot length $(L)$, and knot thickness $(T)$ obtained by the knot shape image taken during the knot-pull test versus the stress applied at the time. Neat PP (a, c) and additive-PP (b, d) fibers were drawn to 10 (a, b), 14 (c), and 15 (d) times. The data are superimposed in (e).
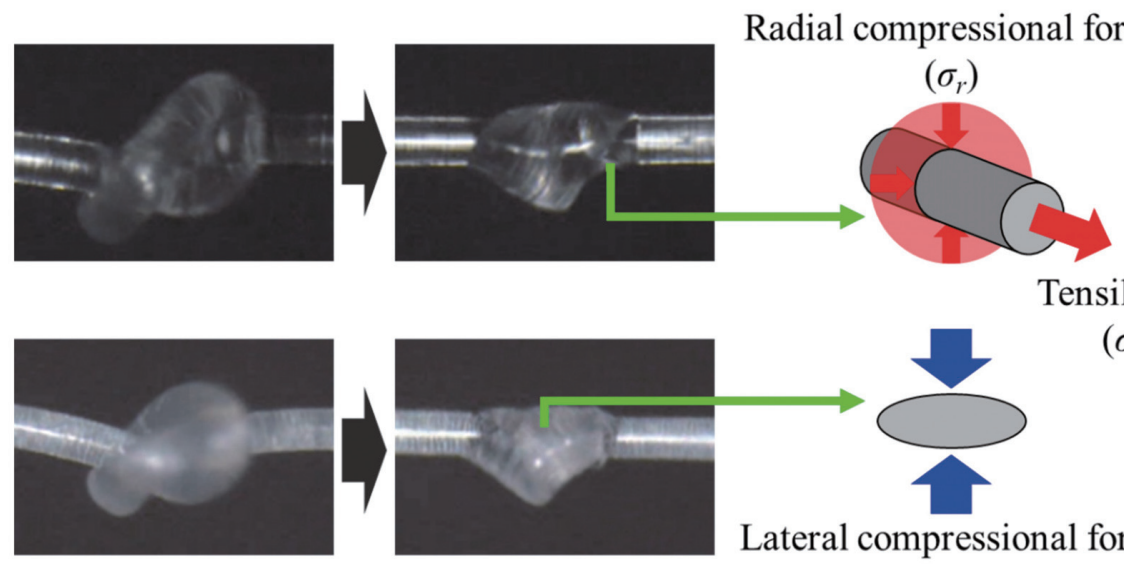

Tensile force

$\left(\sigma_{z z}\right)$

Fig. 10 Typical micro-images taken during the knot-pull test. The images correspond to stresses lower than $100 \mathrm{MPa}$ and those immediately before the break. The low-draw-ratio neat polypropylene (PP) fibers of draw ratio 10 and the high-draw-ratio additive-containing PP fibers of draw ratio 15 are shown in the upper and lower rows, respectively. Major forces applied to the knot are also illustrated.

of the knot entrance as a result of the tensile force, whereas the high-draw-ratio fibers broke at the buckled bending part in the knot with fibrillation as a result of the lateral compressional force. In contrast, the contribution of twisting and shearing forces to knot breakage was limited, as observed in the SEM images.

The stress field of the knotted fiber is expressed by equation (4). As shown in the equation, the tensile stress $\left(\sigma_{z z}\right)$ and radial compressional stress $\left(-\sigma_{r}\right)$ arise normal to the stress difference $\sigma_{z z}+\sigma_{r}$ and hydrostatic pressure $\left(\sigma_{z z}-\sigma_{r}\right) / 3$.

$$
\begin{aligned}
\left(\begin{array}{ccc}
-\sigma_{r} & 0 & 0 \\
0 & -\sigma_{r} & 0 \\
0 & 0 & \sigma_{z z}
\end{array}\right)= & \frac{1}{3}\left\{\left(\begin{array}{ccc}
-\sigma_{z z}-2 \sigma_{r} & 0 & 0 \\
0 & -\sigma_{z z}-2 \sigma_{r} & 0 \\
0 & 0 & 2 \sigma_{z z}+\sigma_{r}
\end{array}\right)\right. \\
& \left.+\left(\sigma_{z z}-\sigma_{r}\right)\left(\begin{array}{ccc}
1 & 0 & 0 \\
0 & 1 & 0 \\
0 & 0 & 1
\end{array}\right)\right\}
\end{aligned}
$$

The tensile breaking stress of the knotted fiber should be lowered by the tensile strength as much as the radial compressional force $\sigma_{r}$. In contrast, the fibrillation of the knotted fiber should be suppressed by the hydrostatic pressure $\sigma_{r} / 3$ compared with that occurring in the loop test. In addition, the buckling and fibrillation are suppressed by fiber friction, which reduces the lateral compressional force. 
The low-draw-ratio fibers had larger loop strengths than tensile strengths. This indicates that the fiber in the knot did not break by buckling but broke because of the tensile force. This tensile breakage was promoted by the increase in the normal stress difference by as much as $\sigma_{r}$. The normal stress difference is highest at the knot entrance where the tensile force is not reduced by the frictional force acting between the fibers in the knot. Moreover, the continuous decrease of knot area $A$ indicates the monotonical increase of $\sigma_{r}$ until fiber breakage. Therefore, the low-draw-ratio fiber likely broke as a result of the normal stress difference between $\sigma_{z z}$ and $\sigma_{r}$. Unlike the low-draw-ratio fibers, there was little difference between the loop and tensile strengths in the high-draw-ratio fibers, which indicates that the fibers did not break as a result of the normal stress difference. The high-draw-ratio fibers, which had already whitened, were easily fibrillated, as observed in the broken fiber ends resulting from the loop and knot-pull tests that were fibrillated. The fiber breakage within the knots also suggested fiber breakage at the buckled part. The decrease of only $T$, as described in Section 3.3, also confirmed that the deformation was concentrated in the buckled bending part before breakage. Therefore, the high-draw-ratio fibers likely broke at the buckled part in the knot with fibrillation because of lateral compressional force.

The effect of the additive was observed in the broken fiber ends, particularly those from the loop test. The broken ends of the additive-containing fibers were fibrillated regardless of the draw ratio. This suggests that the additive promoted fibrillation. Fibrillation was further promoted in the high-drawratio fibers. The fibrillation promotion effect of the additive explains the relatively low knot-pull strength of the additive containing high-draw-ratio fibers, despite their higher tensile strength. The fibrillation promotion effect also explains the relatively low tensile strength of the additive containing low-drawratio fibers, which could be ascertained by their fibrillated broken ends, as observed via SEM. Nevertheless, the additive did not reduce the knotpull strength of the low-draw-ratio fibers because the increase in hydrostatic pressure by as much as $\sigma_{r} / 3$ likely cancelled the fibrillation promotion effect.

\section{Conclusion}

To investigate the breaking mechanism of knotted PP monofilament, that is, thick single fiber, the knot-pull, tensile, and loop strengths were evaluated for neat and additive-containing PP fibers fabricated by melt-spinning and drawing. The broken fiber ends resulting from the loop and knot-pull tests were examined via SEM. The deformation behavior of the knot during the knot-pull test was also investigated.

The knot-pull strength was constantly lower than the tensile and loop strengths. In contrast, the loop strength of the low-draw-ratio fibers was clearly larger than the tensile strength, but largely decreased in the high-draw-ratio fibers. The SEM revealed two forms of broken fiber ends: the low-draw-ratio fibers, which were broken at the knot entrance, hardly produced buckled fiber ends, whereas the high-drawratio fibers, which were broken within the knot, produced buckled and fibrillated fiber ends. The tendency observed for the high-draw-ratio fibers was confirmed by image analysis of the video obtained during the knot-pull test. That is, the knot thickness of the high-draw-ratio fiber continued to decrease until fiber breakage, even after the knot length stopped decreasing. This indicates that fiber buckling occurred before fiber breakage. Therefore, the lowdraw-ratio fiber likely broke as a result of the normal stress difference, whereas the high-draw-ratio fiber probably broke at the buckled part in the knot with fibrillation as a result of lateral compressional force.

The additive increased the tensile strength, particularly in the high-draw-ratio fibers, whereas it barely affected the knot-pull strength. The additive probably leveled the stress applied to the fibrils, but it also promoted fibrillation in the fiber. The knot-pull strength hardly increased because the promotion effect was likely cancelled by the tensile strength increase. The promotion effect also reduced the tensile strength of the low-draw-ratio fibers, but the knot-pull strength was not reduced. This was because fibrillation seemed to be suppressed by the increase in hydrostatic pressure.

\section{Acknowledgments}

The authors would like to thank Mitsubishi Chemical Corporation for kindly supplying the source polymer and additive used in the present study. We also thank Frank Kitching, MSc., from Edanz Group (https://en-author-services.edanzgroup.com/ac) for editing a draft of this manuscript. The study was 
supported by a Grant-in-Aid from the Shinshu University Advanced Leading Graduate Program, and by JSPS KAKENHI (grant numbers JP16K05910 and JP19K05597).

\section{References}

1. K. Yamaki, Journal of the Textile Machinary Society, 18, 356 (1965).

2. Y. Hori, Kobunshi, 11, 150 (1962).

3. H. Yabe, Journal of Chugoku Junior College, 18, 1 (1987).

4. H. Endo, S. Ohira, Sen'i Gakkaishi, 47, 333 (1991).

5. R. Oono, Journal of Home Economics of Japan, 47, 461 (1996).

6. H. Endo, S. Ohira, "Seni binran 3rd edition", Sen”i Gakkai Ed., Maruzen Publishing, 55 (2004) ISBN 978-4-621-07485-5.

7. P. Pieranski, S. Kasas, G. Dietler, J. Dubochet, A. Stasiak, New J. Phys., 3, 10.1 (2001).

8. H. Uehara, H. Kimura, A. Aoyama, T. Yamanobe, T. Komoto, New J. Phys., 9, 65 (2007).
9. C. W. Ashley, “The Ashley Book of Knots”, Faber and Faber, 17 (1944). ISBN 978-0571096596

10. A. Konda, C. Koyama, T. Agatsuma, Bulletin of the Textile Research Institute, 62, 25 (1962).

11. A. Konda, S. Sekiguchi, T. Misaizu, K. Shirakashi, Sen'i Kikai Gakkaishi. 27, T127 (1974).

12. A. J. Uddin, J. Araki, Y. Gotoh, Biomacromolecules, 12, 617 (2011).

13. P. J. Lemstra, R. Kirschbaum, T. Ohta, H. Yasuda, “Developments in Oriented Polyerms-2", I. M. Ward Ed., Elsevier applied science, 69 (1987). ISBN 9781-85166-045-2

14. Y. Atarashi, Sen’i Gakkaishi. 27, 204 (1971).

15. T. Ono, Journal of The Adhesion Society of Japan. 52, 342 (2016).

16. H. Kishi, Y. Kunimitsu, J. Imade, S. Oshita, Y. Morishita, M. Asada, Polymer, 52, 760 (2011).

17. KURARITY ${ }^{\mathrm{TM}}$ Acrylic Block Copolymer Technical information https://www.kuraray.com / uploads /5e4f6cdf20d15/Kurarity_technical_ brochure_en.pdf. (accessed 15 June 2020)

18. T. Kunimitsu, K. Toyoda, T. Ikaga, K. H. Kim, Y. Ohkoshi, K. Koike, Polymer, 202, 122654 (2020). 